\title{
Spontaneous acute type $A$ aortic dissection as a result of autoimmune aortitis without previous aortic dilatation in a 43-year-old man
}

\author{
Peter J. Oberwalder, MD, Gernot Tilz, MD, and Bruno Rigler, MD, Graz, Austria
}

T he most frequent causes of aortic dissection are medial degenerative disease and hypertension. Inflammation and immunopathologic conditions are rare and generally of a single origin. We report here on an unusual case of combined immunopathologic conditions in which simultaneous autoantibodies to nuclear factors, glomerular basement membranes, and neutrophils in accordance with concomitant systemic lupus erythematosus, Wegner disease, and Goodpasture syndrome combined to cause spontaneous acute aortic dissection.

\section{Clinical Summary}

A 43-year-old Caucasian man had a sudden onset of severe chest pain radiating to the neck and interscapular region. Electrocardiographic and biochemical markers showed no evidence of myocardial infarction. The patient's history was unremarkable for hypertension, aneurysmal disease, or any kind of high-risk profile. Immediate computed tomographic scan revealed dissection of the ascending aorta, with no involvement of the remainder of the aorta. Acute surgical repair was performed with deep hypothermic circulatory arrest. The diseased aortic segment was resected, and a hemiarch replacement with a 30-mm woven Dacron polyester fabric tube graft in the supracoronary position was carried out. The patient was extubated 8 hours after the operation and was dis-

From the University Hospital Graz, Department of Cardiac Surgery, Clinical Immunology, and Jean Dausset Laboratory, Graz, Austria.

Received for publication June 24, 2002; accepted for publication July 10, 2002.

Address for reprints: Peter J. Oberwalder, MD, University Hospital Graz, Cardiac Surgery, Auenbruggerplatz 29, Graz A-8036, Austria (E-mail: peter.obw@aon.at).

J Thorac Cardiovasc Surg 2003;125:413

Copyright $\odot 2003$ by The American Association for Thoracic Surgery

$0022-5223 / 2003 \$ 30.00+0$

doi: $10.1067 / \mathrm{mtc} .2003 .132$
TABLE 1. Profiles of abnormal antibody titers found at time of operation

\begin{tabular}{lcc}
\hline & $\begin{array}{c}\text { Patient } \\
\text { value }\end{array}$ & $\begin{array}{c}\text { Reference } \\
\text { value }\end{array}$ \\
\hline Double-stranded DNA (IU/mL) & 440 & $>55$ \\
Cardiolipin (IU/mL) & 71 & $>18$ \\
Nuclear factor GMb (IU/mL) & 10.4 & $>5$ \\
Myeloperoxidase antineutrophil & 85 & $>5$ \\
$\quad \begin{array}{l}\text { cytoplasm antibody (IU) } \\
\text { Proteinase 3 antineutrophil }\end{array}$ & 86 & $>5$ \\
$\quad$ cytoplasm antibody (IU) & & \\
\hline
\end{tabular}

charged from the hospital 8 days later. The further course was uneventful, and at 2 years of follow-up the patient is symptom free, with no evidence of recurrent dissection or aortic dilatation.

Histopathologic examination of the excised aortic wall showed intense infiltration of inflammatory cells within the entire media, pronounced around the vasa vasorum. The cellular infiltrate was composed of lymphocytes, plasma cells, and a mixture of histiocytes and eosinophils. Results of immunopathologic study are listed in Table 1.

\section{Discussion}

Noninfective aortitis is usually associated with a variety of systemic diseases including giant cell arteritis, juvenile rheumatoid arteritis, ankylosing spondylitis, Reiter syndrome, and Behçet disease. Isolated cases of aortitis have been described in patients with systemic lupus erythematosus, scleroderma, rheumatic fever, and Kawasaki syndrome. Because of medial destruction of the aortic wall with disruption and depletion of elastic fibers, dilatation of the ascending aorta occurs. This is distinct from the pattern in our case, in which histologic examination of the aorta showed evidence of combined inflammation. To our knowledge there have been no previous reports of acute dissection without aortic dilatation of an autoimmune nature. 